\title{
Successively expressed cuticular protein genes at the prepupal stage in wing discs of Bombyx mori
}

\author{
Md Saheb Ali ${ }^{1,2^{*}}$, Birendra Mishra ${ }^{3}$, Ahsanul Haque Swapon ${ }^{4}$ and Masamitsu Yamaguchi ${ }^{1}$
}

\begin{abstract}
Background: We classified cuticular protein genes expressed at prepupal stage in wing discs of Bombyx mori into six groups according to their developmental expression and ecdysone responsiveness. Their expression pattern is suggested to be regulated by ecdysone-responsive transcription factors, whose transcripts showed resemblance with those of cuticular protein gene expression.

Result: Group1 and Group2 CP genes showed peak expression at stage W2. Group3 CP genes showed high expression at stage W3E and W3L and were upregulated by 20E addition, showing a peak $12 \mathrm{~h}$ after $20 \mathrm{E}$ pulse treatment. Group4 CP gene transcripts started expression from stage V5 and peaked at stage W3L. Some genes showed significant increase 4 or $6 \mathrm{~h}$ after 20E addition and were induced $6 \mathrm{~h}$ and showed a peak $18 \mathrm{~h}$ after the $20 \mathrm{E}$ pulse treatment. Group5 CP gene transcripts peaked at the same stage W3L. Some Group5 genes showed significant increase $6 \mathrm{~h}$ after the 20E addition, while others were not induced by the $20 \mathrm{E}$ addition. These different sub-groups showed different expression profiles in the feeding stage. Transcripts of this group genes were induced $12 \mathrm{~h}$ and showed a peak $18 \mathrm{~h}$ after the 20E pulse treatment. Group6 CP genes peaked at the stage P0, were not induced by the $20 \mathrm{E}$ addition, and showed a peak $24 \mathrm{~h}$ after the 20E pulse treatment. Group3, 4, 5, and 6 CP genes are suggested to be regulated by BHR4, BR-C, E74A, and BFTZ-F1, respectively. ERTFs showed different responsiveness to 20E concentration. BR-C was most and E74A was least insensitive. The addition of cycloheximide inhibited BR-C, E74A, and BFTZ-F1 expression depending on the length of treatment after ecdysone pulse treatment, which suggests that BHR4 induced BR-C, E74A, and BFTZ-F1.
\end{abstract}

Conclusion: Expression patterns of CPS were determined by the ecdysone-responsiveness and the related ERTFs expressed in the prepupal stage in B. mori wing discs.

Keywords: Cuticular protein genes, Ecdysone, Silkworm, ERTF, Wing disc

\section{Background}

Approximately two hundred $\mathrm{CP}$ genes have been recognized in Anopheles gambiae (Cornman et al., 2008) and Bombyx mori (Futahashi et al., 2008), and a number of distinct families of CPs have been documented (Willis, 2010). Three types of CPRs have been reported, RR1 is a soft and RR2 is a hard cuticular protein (Andersen, 1998; Willis, 2010), and RR3 is composed of few groups (Andersen,

\footnotetext{
*Correspondence: alibjri@gmail.com; sahebbjri@yahoo.com

'Department of Applied Biology, Kyoto Institute of Technology, Sakyo-ku, Kyoto 606-8585, Japan

${ }^{2}$ Faculty of Agriculture, Bangladesh Jute Research Institute, Manik Mia

Avenue, Dhaka 1207, Bangladesh

Full list of author information is available at the end of the article
}

2000; Willis, 2010). Other families of cuticular protein gene were also identified in the genome of B. mori. CPF and CPFL are derived from their fifty-one amino acids (CPF) and their sequence similarity with CPF (CPFL) (Andersen, Rafn, \& Roepstorff, 1997; Togawa, Dunn, Emmons, \& Willis, 2007; Togawa, Natkato, \& Izumi, 2004). Binding with chitin was not observed in the case of CPF and CPFL (Togawa et al., 2007), and whether they are used to form epi-cuticle or exo-cuticle remains to be solved. CPT family is named from the mutation of body shape (TweedleD, Guan, Middlebrooks, Alexander, \& Wasserman, 2006), and CPG is named from high content of glycine. $\mathrm{CPH}$ (cuticular protein hypothetical) family is putative cuticular proteins (Futahashi et al., 2008). They 
were found in Bombyx genome, and they do not have R\&R consensus sequence. A number of cuticular protein genes appeared during insect evolution, which makes it possible to construct different types of cuticle in different stages and spaces. Nevertheless, the mechanism determining the combination of cuticular proteins has not been clarified.

Generally the epicuticle and the procuticle are the cuticular layers in the insect and later secreted to thick exo-cuticle and then endo-cuticle (Wigglesworth, 1972) and constructed by chitin and cuticle proteins with R\&R consensus, and other types of cuticular protein constructed three cuticle layers. Correspondent with this, the clustering $\mathrm{CP}$ genes expressed around the molting stage were observed in B. mori (Okamoto, Futahashi, Kojima, Mita, \& Fujiwara, 2008; Liang, Zhang, Xiang, \& He, 2010; Ali, Iwanaga, \& Kawasaki, 2013; Ali, Rahman, \& Swapon, 2015; Ali, Mishra, Rahman, \& Swapon, 2016; Ali et al., 2016; Ali, Hossain, \& Mishra, 2018) and A. gambiae (Togawa et al. 2008). Charles (2010) described about the regulation of expression of insect $\mathrm{CP}$ genes. However, the mechanism of the successive $\mathrm{CP}$ production has not been clear.

Recently, we clarified the regulation of $\mathrm{CP}$ genes by several ERTFs in Bombyx wing discs as follows. Direct regulation by EcR/USP was also observed in the expression of $\mathrm{CP}$ gene, BmorCPR21 (previously classified as BMWCP10), where BR-C functioned together with EcR/USP (Wang, Moriyama, Iwanaga, \& Kawasaki, 2010). BmorCPR99 (previously classified as BMWCP2) and BmorCPR92 (previously classified as $B M W C P 5$ ) transcripts were induced by an ecdysone pulse through an ecdyson-responsive transcription factor (ERTF), $\beta F T Z-F 1$, which bound to the upstream region of these $\mathrm{CP}$ genes and increased their promoter activity (Nita et al., 2009; Wang, Nita, Iwanaga, \& Kawasaki, 2009). Expression of BmorCPR93 (previously classified as BMWCP4; Ali, Iwanaga, \& Kawasaki, 2012) and BmorCPR23 (Ali et al., 2013), were regulated by E74A, and BmorCPH5 was by BHR3 (Ali et al., 2013). Thus, CP genes that are the final targets of ecdysone signaling became key players to clarify the mechanism of ecdysone signaling function.

Ecdysteroid surge brings about insect metamorphosis. The successive ERTF expression by the ecdysteroid secretion, which is called as ecdysone signaling cascade, has been studied in Dipteran and Lepidopteran insect (Dittmer et al., 2015; Elgendy, Tufail, Mohamed, \& Takeda, 2019; Hiruma \& Riddiford, 2001; King-Jones, Charles, Lam, \& Thummel, 2005; Lam, Jiang, \& Thummel, 1997; Pan et al., 2018; Riddiford, Hiruma, Zhou, \& Nelson, 2003; Riddiford \& Truman, 1993; Sekimoto, Iwami, \& Sakurai, 2006; Thummel \& S., 1995; Zhao et al., 2017). Before ecdysteroid surge in the hemolymph, $E 74 B$ and $B R-C$ transcripts are induced (Sekimoto et al.,
2006; Sekimoto, Iwami, \& Sakurai, 2007) in the low concentration of the hemolymph ecdysteroid. By the expression of BR-C, commitment to pupa occurs (Koyama, Syropyatova, \& Riddiford, 2008; Zhou \& Riddiford, 2001) and through the function of these ERTFs, insect can prepare for pupation (Fletcher \& Thummel, 1995; Konopova \& Jindra, 2008; Parthasarathy, Tan, Bai, \& Palli, 2008). After ecdysteroid surge, DHR3, DHR4, E75A, E75B, E74A and BFTZ-F1 are successively induced in Drosophila melanogaster (Huet, Ruiz, \& Richards, 1995). Homologs of these ERTFs were isolated, and the functions of them have been reported (Hiruma \& Riddiford, 2001; Stilwell et al., 2003; Sun, Hirose, \& Ueda, 1994; Sun, Zhu, Chen, \& Raikhel, 2005; Zhou \& Riddiford, 2001). Among them, DHR3 have been known as a main factor bringing about insect metamorphosis through the regulation of other following factors (King-Jones et al., 2005; Lam et al., 1997; White, Hurban, Watanabe, \& Hogness, 1997) but the interactions of ERTFs are not yet clarified.

Not so many target genes of these ERTFs are yet identified. In the present study, we tried to clarify how CPs are successively produced to construct pupal cuticle layers. We classified cuticular protein $(\mathrm{CP})$ genes expressed at prepupal stage in wing discs of Bombyx mori into six groups according to their developmental expression, ecdysone responsiveness and regulating ERTFs. Moreover, our wing disc culture system helped the understanding of the interaction of these ERTFs.

\section{Materials and methods}

\section{Experimental animals and developmental stages}

A hybrid strain of $B$. mori was reared at $25^{\circ} \mathrm{C}$ in a $12 \mathrm{~h}$ light:12 h dark photoperiod. Under these conditions larvae started wandering on day six of the fifth larval instar, pupation occurred 3 days thereafter, and adults eclosed 10 days after pupation. The periods (in days) corresponding to the developmental stages of the fourth to fifth larval ecdysis, wandering, pupation, and eclosion were designated as $\mathrm{V} 0$, W0, $\mathrm{P} 0$, and $\mathrm{A} 0$, respectively. The three days before pupation were designed as W1W3. The W3 stage was divided into three different substages, W3 early (W3E), W3 mid (W3M), and W3 late (W3L). The W3 sub-stages were determined on the time and visible shortening of the length of the leg.

\section{In vitro culture of wing discs}

Wing discs of larvae at the V4 and W2 stages were prepared for the in vitro culture. For wing disc preparation, the fat body and trachea were carefully removed under a microscope. The culture was carried out according to a previous report (Kawasaki, 1989) at $25^{\circ} \mathrm{C}$ under sterile conditions. We conducted in vitro induction at various times following administration of $2 \mu \mathrm{g} / \mathrm{ml} 20 \mathrm{E}$ to $\mathrm{V} 4$ wing discs and after cessation of a $12 \mathrm{~h}$ pulse of $2 \mu \mathrm{g} / \mathrm{ml}$ 
$20 \mathrm{E}$ to discs from W2. The necessity of protein synthesis for induction was tested in the cultured discs by administration of $50 \mu \mathrm{g} / \mathrm{ml}$ cycloheximide from the start of culture (V4) or at the time of 20E removal (W2).

\section{RNA sample preparation and first-strand CDNA synthesis}

To determine the expression levels of the $\mathrm{CP}$ genes and transcription factors, total RNA were extracted at distinct stages from wing discs using RNAiso (Takara, Japan) and quantified by spectrophotometry at $260 \mathrm{~nm}$. First-strand cDNA was synthesized from $1 \mu \mathrm{g}$ total RNA in a $10 \mu \mathrm{l}$ reaction mixture using ReverTra Ace (Toyobo, Japan).

\section{Quantitative RT-PCR}

qRT-PCR was conducted on an ABI7500 real-time PCR machine (Applied Biosystems) using the FastStart Universal SYBR Green Master (Roche). Each amplification reaction was performed in a $25 \mu \mathrm{l}$ qRT-PCR reaction under the following conditions: denaturation at $95^{\circ} \mathrm{C}$ for $10 \mathrm{~min}$ followed by 40 cycles of treatment at $95^{\circ} \mathrm{C}$ for $10 \mathrm{~s}$ and at $60^{\circ} \mathrm{C}$ for $1 \mathrm{~min}$. Ribosomal protein S4 (Bmrpl:GenBank accession no. NM_001043792) was used as a control gene. The data were normalized by determination of the amount of Bmrpl in each sample to eliminate variations in mRNA and cDNA quality and quantity. The transcript abundance value of each individual was the mean of three replicates. Each pair of primers was designed using Primer3 software (http://frodo.wi.mit.edu/). The gene specific primers are listed in Additional file 2: Table S1.

\section{BLAST search of genomic sequences of cuticular protein genes}

The cDNA sequences were used for BLAST search analysis to obtain their upstream sequences. BLAST search was carried out using the genomic database of $B$. mori (http://kaikoblast.dna.affrc.go.jp/).

\section{Results}

$\mathrm{CP}$ genes expressed in prepupal stage show six distinctive developmental patterns

We compared the expression pattern of $\mathrm{CP}$ genes expressed prepupal stage to clarify how CPs are successively produced to construct pupal cuticle layers. We found six different expression profiles of these $\mathrm{CP}$ genes. We have reported that the induction of successively produced CPs was regulated by successively appeared ERTFs as indicated in Fig. 1. We classified CPs into six groups depending on the stage of the peak appearance and the ecdysone responsiveness.

We named Group1 to Group6 according to the stage of expression peak. BmorCPR21 only belongs to Group1, and the gene of Group1 was directly induced by $20 \mathrm{E}$,
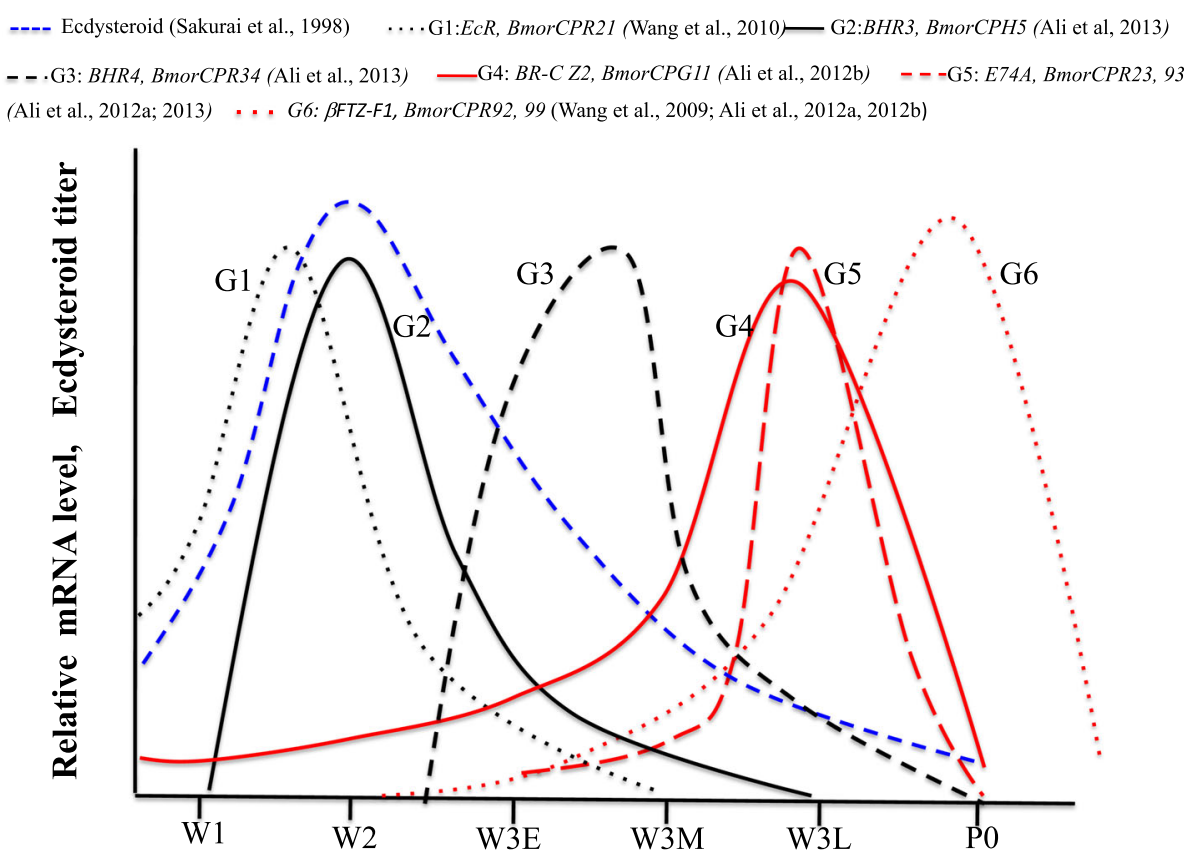

Developmental stage

Fig. 1 Schematic representation of cuticular protein genes and related ecdysone-responsive transcription factors in parentheses expressed in wing discs of B. mori in the late fifth larval instar. Ecdysteroid titer (Sakurai, Kaya, \& Satake, 1998), BmorCPR21 (Wang, Iwanaga, \& Kawasaki, 2009), BmorCPR92 (Wang, Nita, et al., 2009), BmorCPR99, BmorCPH5, BmorCPR93 (Ali, Iwanaga, \& Kawasaki, 2012) and BmorCPG11 (Ali, Wang, Iwanaga, \& Kawasaki, 2012) are indicated 
and its expression increased from the stage W0 and peaked at the stage W2 slightly earlier than Group2 gene. BmorCPH5 only belongs to Group2 and was regulated by BHR3 (Ali, Wang, et al., 2012).

$\mathrm{CP}$ genes that belong to Group3 were BmorCPR34 and BmorCPH33, and BmorCPR34 has been reported to be regulated by BHR4 (Ali, Wang, et al., 2012). Expression pattern of BmorCPH33 resembled that of BmorCPR34 and $B H R 4$, and its transcripts increased from the stage $\mathrm{W} 3 \mathrm{E}$ and showed broad expression peak at the stage W3E to W3L (Additional file 1: Figure S1).

Expression pattern of Group4 $\mathrm{CP}$ genes resembled BmorCPG11 and BR-C Z2. They increased from W0 stage, continued increase until W3L peak (Additional file 1: Figure S2). Ecdysteroid titer in the hemolymph started to increase around W0, and BR-C is inducible by the low concentration of 20E (Chen, Zhu, Sun, \& Raikhel, 2004; Muramatsu, Kinjoh, Shinoda, \& Hiruma, 2008; Sekimoto et al., 2006), therefore it is suggested that BR-C expression is observed from around W0. BmorCPG12, BmorCPG13, BmorCPG16, BmorCPG17, BmorCPG24, and BmorCPH30 belong to this group. The expression pattern of these $\mathrm{CP}$ genes resembled BmorCPG11 (Ali, Wang, et al., 2012) that was regulated by BR-C.

Expression pattern of Group5 $\mathrm{CP}$ genes resembled that of E74A and BmorCPR93 that was regulated by E74A (Ali, Wang, et al., 2012) and showed expression peak at the stage W3L. Twelve CP genes belonged to this group (Additional file 3: Table S2, Additional file 1: Figure S3). Transcripts of $\mathrm{CP}$ genes of this group peaked at the stage W3L, but the expression pattern was different in different $\mathrm{CP}$ genes. Transcripts of BmorCPT3 were detected from the stage W3E, while BmorCPR42, BmorCPR122, and $B$ morCPR124 transcripts were barely detected except the stage W3L, and other CP genes showed expression at the stages W3M and P0.

Expression pattern of Group6 $\mathrm{CP}$ genes resembled that of BFTZ-F1, BmorCPR45 (Ali, Iwanaga, \& Kawasaki, 2012), BmorCPR92 (Wang, Nita, et al., 2009) and BmorCPR99 (Nita et al., 2009), and these three $\mathrm{CP}$ genes were regulated by $\beta$ FTZ-F1. Transcripts of $23 \mathrm{CP}$ genes peaked at the stage P0. Fourteen CPRs that have R\&R consensus, two CPGs, two CPFLs, three CPHs, and two CPTs were identified in this group. Although most CPR genes showed sharp peak at the stage P0, BmorCPG14, BmorCPFL4, BmorCPH2, and BmorCPH3O showed transcripts from the stage W3M (Additional file 1: Figure S4).

Thus, we classified 53 CPs expressed at prepupal stage into six groups.

\section{$\mathrm{CP}$ genes expressing the same developmental profile showed the same ecdysone-responsiveness}

Group1 gene, BmorCPR21, has been reported to be induced directly by 20E (Wang et al., 2010). Group2 gene,
BmorCPH5, was reported to be induced by $20 \mathrm{E}$ and regulated by BHR3 (Ali et al., 2013). Group3 gene, $\mathrm{BmorCPH} 33$ transcripts were induced by $20 \mathrm{E}$ addition (Additional file 1: Figure S5) as well as BmorCPR34 that has been reported to be regulated by BHR4 (Ali et al., 2013). Group4 genes were upregulated by the addition of $20 \mathrm{E}$, but the response was not uniform (Additional file 1: Figure S6). Thus, Goup1 to Group4 CP genes were upregulated by the $20 \mathrm{E}$ addition.

Four genes were upregulated (Additional file 1: Figure S7) as reported before (BmorCPR93, Ali, Wang, et al., 2012; BmorCPR23, Ali et al., 2013), but five were not by $20 \mathrm{E}$ in Group5. Response to $20 \mathrm{E}$ of upregulated genes showed slow one compared with Group1-4 genes. Unexpectedly, five CP genes of Group5 were not upregulated by the $20 \mathrm{E}$ addition. To clarify the different $20 \mathrm{E}$ response in Group5 CP genes, we examined the developmental expression of Group5 CP genes from the beginning of the fifth larval instar. Interestingly, $\mathrm{CP}$ genes that were upregulated by the $20 \mathrm{E}$ addition did not show transcripts until the stage W2 (Additional file 1: Figure S8). In contrast, $\mathrm{CP}$ genes that were not upregulated by the $20 \mathrm{E}$ addition showed transcripts from the stage V0 to V5 (Additional file 1: Figure S9). We used wing discs of the V4 stage, when the hemolymph ecdysteroid was barely detected, for the experiment of $20 \mathrm{E}$ addition. From these results, $\mathrm{CP}$ genes that did not respond to $20 \mathrm{E}$ addition were considered to be regulated by other factor in wing discs in the feeding stage. Therefore, the response to $20 \mathrm{E}$ was different in these $\mathrm{CP}$ genes from those of $20 \mathrm{E}$ responsive genes that were not induced in the feeding stage.

Group6 CP genes were not induced by $20 \mathrm{E}$ addition (data not shown) as reported before with BmorCPR45 (Ali et al., 2012a), BmorCPR92 (Wang, Nita, et al., 2009) and BmorCPR99 (Nita et al., 2009).

Thus, CP genes in different groups showed different response to 20E addition in wing discs of the V4 stage.

\section{CP genes belonging to Group3, Group4, Group5, and Group6 were induced by the ecdysone pulse treatment} Group3 gene, BmorCPH33 transcripts were induced in $6 \mathrm{~h}$ and peaked at $12 \mathrm{~h}$ by the 20E pulse treatment (Additional file 1: Figure S10). Transcription of Group4 genes was induced by the $20 \mathrm{E}$ pulse treatment. Increase was observed in $6 \mathrm{~h}$ in every gene, and they peaked at $18 \mathrm{~h}$ after the 20E removal (Additional file 1: Figure S11). Interestingly, the increasing patterns of these expressions resembled expression profiles of developmental expression. All the genes in Group5 were induced by the $20 \mathrm{E}$ pulse, and most of them increased from $12 \mathrm{~h}$ and peaked $18 \mathrm{~h}$ after the 20E removal (Additional file 1: Figure S12). All the genes in Group6 were induced, and most of them peaked at $24 \mathrm{~h}$ by the $20 \mathrm{E}$ pulse (Additional file 
1: Figure S13). CP genes of this Group showed different peak from other two Groups. Their peaks were observed later than other two groups as observed in vivo peaks. Their peaks in vivo were at the stage P0, while those of Group4 and Group5 were the stage W3L.

Surprisingly to say that expression profiles of Group6 CP genes after ecdysone pulse treatment showed similar pattern to those of developmental profiles (Additional file 1: Figure S13). BmorCPR4, BmorCPR10, BmorCPR75, BmorCPG4, and BmorCPH1 showed sharp peaks after 24h, and BmorCPFL4 and BmorCPH31 showed broad peaks at 24 h. Group5 genes, BmorCPR3, BmorCPR42, BmorCPR95 (previously classified as BMWCP3), BmorCPR104 (previously classified as BMWCP1), BmorCPR122 (previously classified as BMWCP8) and BmorCPR124 (previously classified as $B M W C P 7)$, and $B m o r C P H 18$ showed peaks at 18 $\mathrm{h}$, which is similar to developmental expression profiles. In contrast, BmorCPG12, BmorCPG13, and BmorCPH30 showed low level of transcripts at the time of ecdysone removal, which is similar to the transcripts level of W2 stage in developmental stage. Their expression profiles resemble those of the developmental stage. The developmental profiles of $\mathrm{CP}$ genes seem to reflect the ecdysone responsiveness of each $\mathrm{CP}$ genes.

\section{The relatedness between the clustering of $\mathrm{CP}$ genes in} the genome and their expression profiles

We did blast search to clarify the relationship between the clustering of $\mathrm{CP}$ genes on the chromosome and the expression profiles of $\mathrm{CP}$ genes. We found two clusters of $\mathrm{CP}$ genes that are expressed in prepupal stage. Two clusters exist in $3 \mathrm{Mb}$ on Chromosome22 (Fig. 2). All the $\mathrm{CP}$ genes belonging to Cluster I are RR1 genes, including one gene of Group3, four genes of Group5, and one gene of Group6. In contrast, all the $\mathrm{CP}$ genes belonging to Cluster II are RR2 genes, including five genes of Group5 and five genes of Group6. The result showed that Cluster I contained $\mathrm{CP}$ genes expressed at the stage W3M, W3L and P0 and Cluster I contained CP genes expressed at the stage $\mathrm{W} 3 \mathrm{~L}$ and $\mathrm{P} 0$, which suggests that chromosome would be loosened and transcription factors are easy to access their binding sites during these stages, not strictly in a short time.

\section{Difference of ERTFs responsiveness to ecdysone}

Thus, the expression of CP genes is suggested to be regulated by ERTF that showed similar developmental expression pattern with correspondent $\mathrm{CP}$ genes. Therefore, the timing of $\mathrm{CP}$ gene expression is suggested to be determined by the related ERTF, which let us examine the mechanism of the determination of ERTF-expression timing.

The first, we examined the response to different concentration of $20 \mathrm{E}$ addition. $B R-C$ responded to low concentration, and BHR3 and BHR4 responded to $0.2 \mu \mathrm{g} / \mathrm{ml}$ 20E, while $E 74 A$ responded to 2 but not below $0.2 \mu \mathrm{g} / \mathrm{ml}$ (Fig. 3). Expression of $B R-C$ in vivo reflected its responsiveness to 20E and was expressed from V5 stage (Ali, Iwanaga, \& Kawasaki, 2012) when ecdysteroid titer in the hemolymph was low (Sakurai et al., 1998). Therefore, it is suggested that $B R-C$ transcripts increased from the stage V5 and were not inhibited by other factors. BHR3 and BHR4 were induced by 0.2 but not by $0.02 \mu \mathrm{g} / \mathrm{ml}$ 20E (Fig. 3). This reflects in vivo induction of BHR3 and

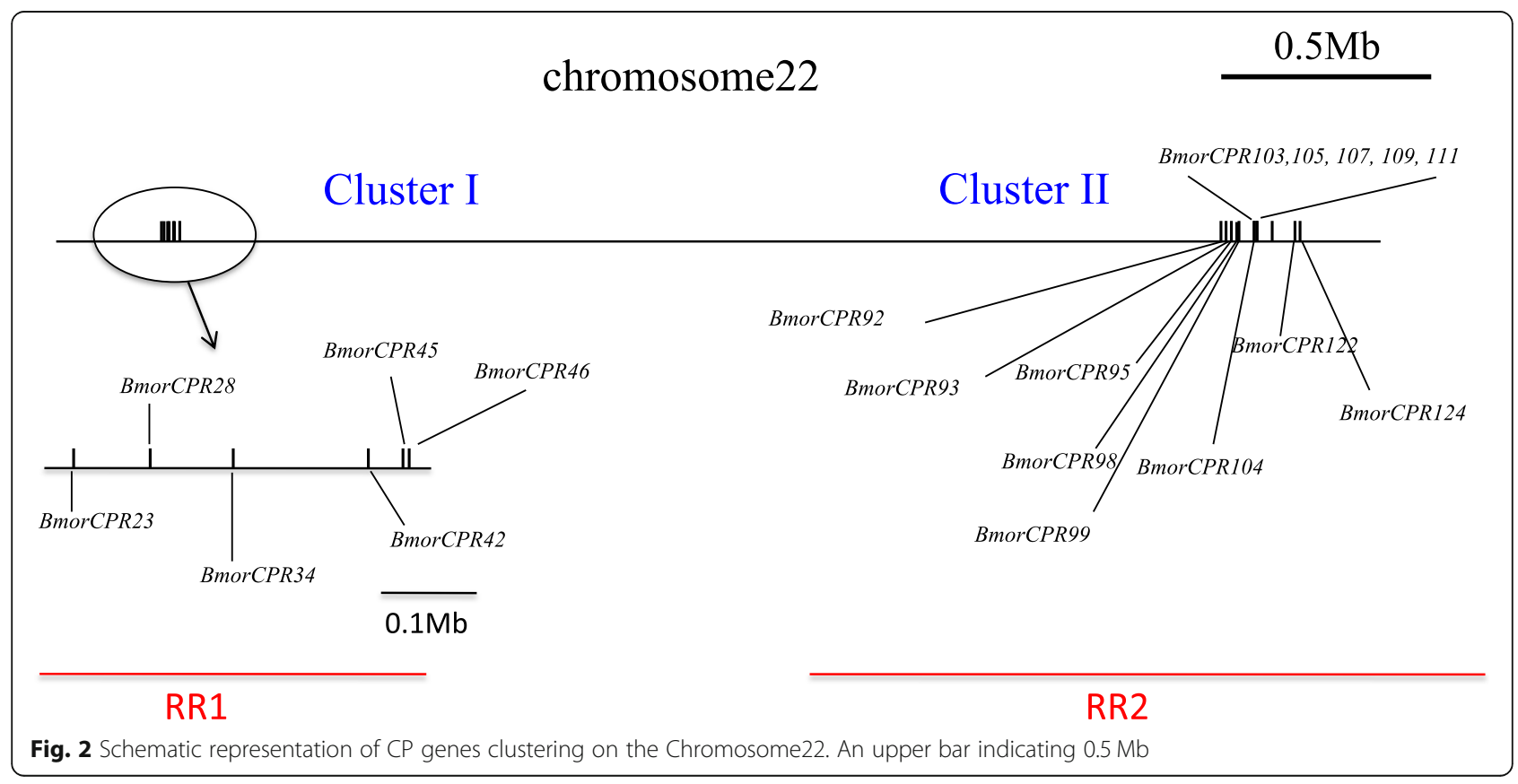




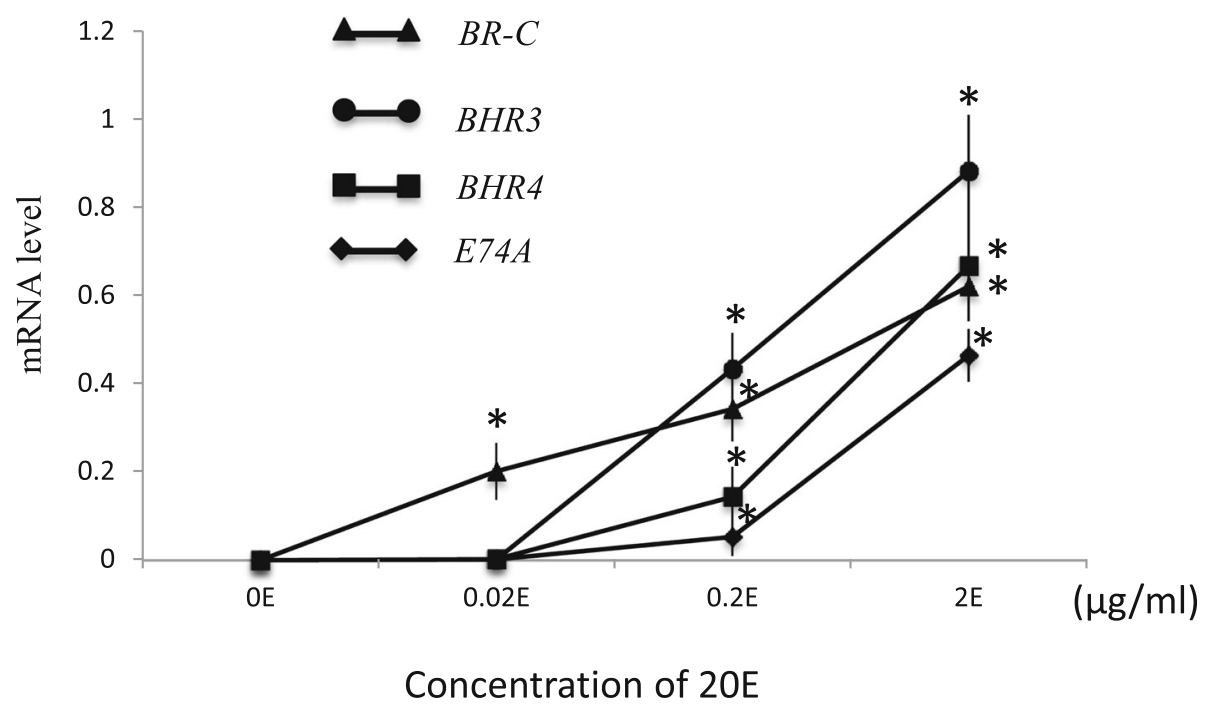

Fig. 3 Induction of transcripts of ERTFs by different concentration of 20E. V4 wing discs were incubated for the indicated time in a medium containing indicated concentration of 20E. RNA was extracted from wing discs and reverse-transcribed to cDNA for use in qRT-PCR. Values were first normalized to $\mathrm{rpl}$ and then plotted relative to the $0 \mathrm{~h}$ value for each panel. Each datum was calculated from three independent experiments. The result is expressed as the mean \pm S. E. M. Asterisks indicate $p<0.05$ significance by the Student's $t$ test. Level of mRNA of the ecdysone treatment

BHR4, since they were not induced before the stage W0 (Ali, Wang, et al., 2012). E74A was induced by highest concentration of 20E. Thus, it is suggested that these CP regulating ERTFs have different responsiveness to $20 \mathrm{E}$ concentrations, which brings about the appearance of these ERTFs, together with the responsiveness to ecdysone pulse as describes below.

\section{BHR3 and BHR4 control the expression of ecdysone-} responsive transcription factors expressed prepupal stage in wing discs of $B$. mori

The effects of cycloheximide treatment were examined to analyze the factors that induced ERTFs after 20E pulse treatment. BHR3 transcripts decreased by the 20E pulse treatment, while BHR4, BR-C and $\beta F T Z-F 1$ transcripts increased from $6 \mathrm{~h}$ and peaked $12 \mathrm{~h}, 18 \mathrm{~h}$ and $24 \mathrm{~h}$ after treatment, respectively. In contrast, E74 transcripts increased from $12 \mathrm{~h}$ and showed sharp peak $18 \mathrm{~h}$ after $20 \mathrm{E}$ removal, as reported by Ali, Wang, et al. (2012). If cycloheximide was added throughout the time after pulse treatment, BHR4, BR-C, E74A, and BFTZ-F1 transcripts were not induced (Fig. 4). In contrast, the cycloheximide treatment of first $12 \mathrm{~h}$ or $6 \mathrm{~h}$ after $20 \mathrm{E}$ removal reduced BR-C, E74A and $\beta F T Z-F 1$ transcripts depending on the length of cycloheximide treatment. In case of BHR3 and BHR4, no effective reduction was observed. From this, it is suggested that BHR4 was translated and induced BR-C, E74A, and $\beta F T Z-F 1$ during the first $6 \mathrm{~h}$ and $12 \mathrm{~h}$.

\section{Discussion}

We classified all the CP genes expressed in the prepupal stage into six groups, depending on the peak timing and ecdysone-responsiveness. They showed peak expression from the stage W2 to P0. Their expression profiles resembled those of corresponding ERTFs.

\section{Prepupal CP genes showed different ecdysone responsiveness, resulted in the different developmental profiles}

Group1 gene, BmorCPR21, has been reported to be regulated by EcR and BR-C Z2 (Wang et al., 2010; Wang, Nita, et al., 2009); EcR bound to EcRE upstream of BmorCPR21. This is the first CP gene that has EcRE, is directly regulated by ecdysone, and showed expression peak at the stage W2 slightly earlier than Group2 gene.

Group2 CP, BmorCPH5, which has been reported to be regulated by BHR3 (Ali, Wang, et al., 2012), induced by the addition of $20 \mathrm{E}$ but decreased by the $20 \mathrm{E}$ withdrawal, showed expression peak at the stage W2. BHR3 and its homolog, DHR3, is a candidate of the factor that brings about insect metamorphosis (Ali, Wang, et al., 2012; King-Jones et al., 2005; Lam et al., 1997; Lam, Bonnie, Bender, \& Thummel, 1999; White et al., 1997). $\mathrm{BmorCPH5}$ is the first $\mathrm{CP}$ gene that is regulated by DHR3 homolog. Thus, our system clarified the function of EcR and BHR3 to induce CP gene expression, but the function of these CPs yet to be clarified in future.

Group3 CP, BmorCPH33, is suggested to be regulated by BHR4, was induced by the $20 \mathrm{E}$ addition and the ecdysone pulse treatment, showed broad expression peak at the stage W3E to W3L, similar to BmorCPR34 has been reported to be regulated by BHR4 (Ali, Wang, et al., 2012). 


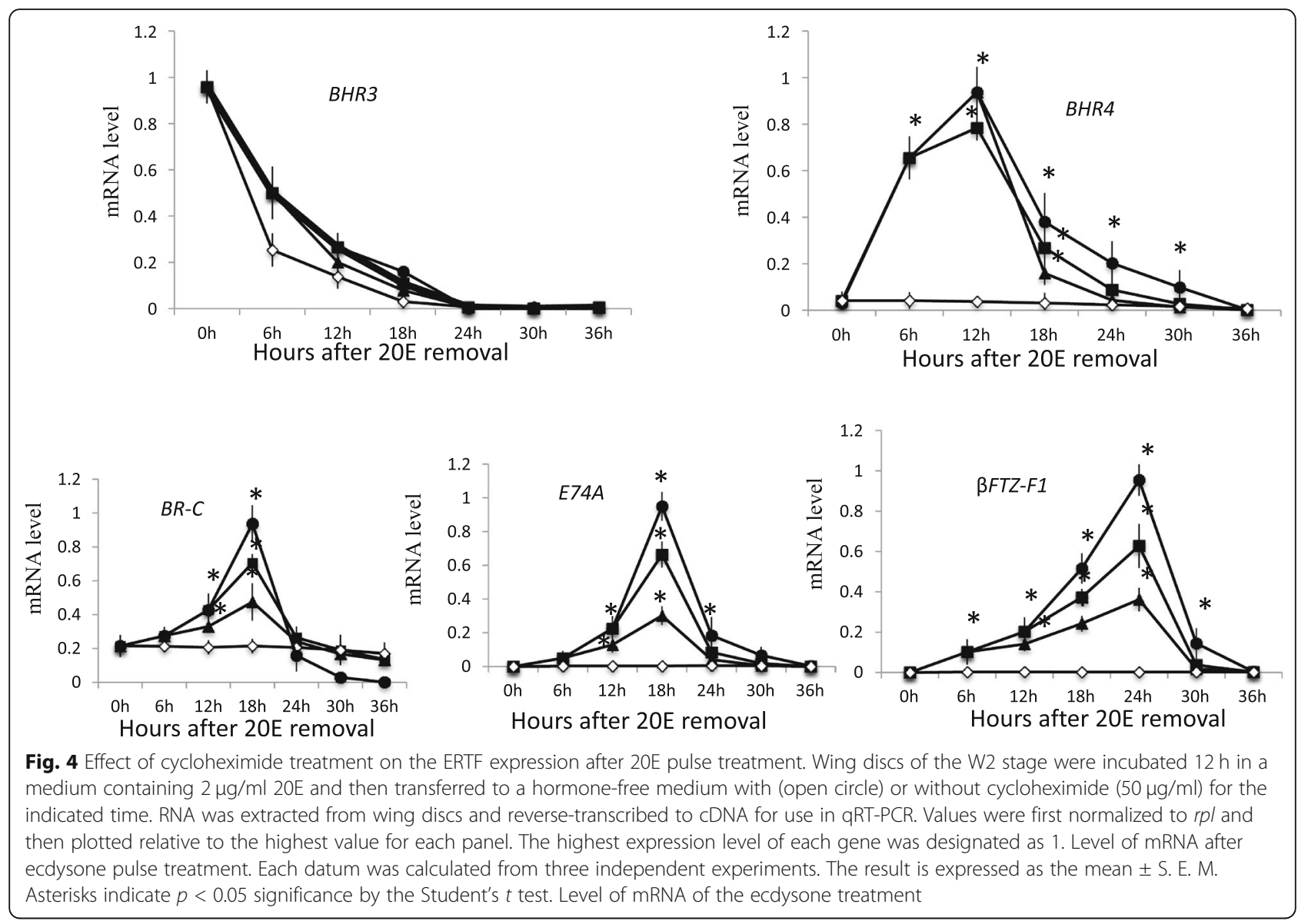

Group4 CPs are, suggested to be regulated by BR-C, induced both by ecdysone and ecdysone pulse, and were induced from the stage V5, peaked at the stage W3L. Different from CPs of other Groups, they were transcribed before wandering stage. This result suggests that the expression of Group4 CPs is not inhibited by other factors. The developmental expression and ecdysone responsiveness of CPs in Group4 resembled those of BmorCPG11 that was regulated by BR-C (Ali, Iwanaga, \& Kawasaki, 2012). BR-C is a key ERTF that determines the pupal character (Bayer, Zhou, Zhou, Riddiford, \& von Kalm, 2003; Uhlirova et al., 2003; Zhou \& Riddiford, 2002), commits insects to the pupa (Muramatsu et al., 2008; Zhou \& Riddiford, 2001), and functioned together with other ERTFs (Chen, O'Keefe, \& Hodgetts, 2002; Wang, Iwanaga, \& Kawasaki, 2009; Wang, Nita, et al., 2009). In spite of these reports, Ali, Wang, et al. (2012) reported that BR-C functioned primarily on the promoter of BmorCPG11, and the expression profile of $\mathrm{CP}$ genes in Group4 resembled BR-C and BmorCPG11. Therefore, it is suggested that in case of CP genes, BR-C functions as a primary factor. Six CPGs and one $\mathrm{CPH}$ belong to this group, and none of them has R\&R consensus. Moreover, their early transcription suggests the involvement of CPs of this group in the epicuticle.
Eighteen CPs belonged to Group5. Five RR1, ten RR2, two $\mathrm{CPH}$, and one CPT genes were identified in this group. All of them showed similar response in the $20 \mathrm{E}$ pulse treatment and developmental expression after wandering stage. Some genes were induced by $20 \mathrm{E}$ addition, but other genes did not respond to 20E in the stage V4 wing discs. The former genes were not expressed in the feeding stage, while latter genes were transcribed in the feeding stage. It is suggested that the difference is response to another factors that regulate expression of CP genes in the feeding stage. The factors would be clarified. CP genes of this group are suggested to be regulated by E74A.

Twenty-four CP genes including six RR1s, nine RR2s, two CPGs, three CPHs, two CPFLs, and two CPTs were identified in Group6. Their developmental expression profiles and ecdysone responsiveness resembled BmorCPR45, BmorCPR92, and BmorCPR99 that have been revealed to be regulated by BFTZ-F1 (Ali, Iwanaga, \& Kawasaki, 2012; Nita et al., 2009; Wang, Nita, et al., 2009). $\beta F T Z-F 1$ is thought to be a key transcription factor at the prepupal stage, and several target genes have been reported (Kawasaki, Hirose, \& Ueda, 2002; Murata, Kageyama, \& Hirose, 1996; Shiomi, Niimi, Imai, \& Yamashita, 2000). 
Thus, six groups of $\mathrm{CP}$ genes are transcribed at prepupal stage and expression is regulated by correspondent ERTFs, which indicate ERTFs determine the final expression stage of CPs. The reason of their sequential expression is each groups of CPs have each regulating ERTFs, respectively as Ali, Wang, et al. (2012) reported.

\section{ERTF appearance and interaction}

E74A rapidly increases after the stage W3M. E74A was induced slightly by $0.2 \mu \mathrm{g} / \mathrm{ml}$ and strongly by $2 \mu \mathrm{g} / \mathrm{ml} 20 \mathrm{E}$, therefore E74A is suggested to be inducible at the stage W2, but it is not in Bombyx larvae. Therefore, it is suggested that the expression of E74A is suppressed by other factor. E74A is suggested to be suppressed by BHR3, as the suppression of E74A by DHR3 (White et al., 1997) was reported in Drosophila. Induction of E74A is inhibited by the addition of cycloheximide $6 \mathrm{~h}$ and $12 \mathrm{~h}$ after $20 \mathrm{E}$ removal, during which BHR4 transcripts increased; therefore, BHR4 is suggested to induce E74A.

Induction of $\beta F T Z-F 1$ is inhibited by the addition of cycloheximide $6 \mathrm{~h}$ and $12 \mathrm{~h}$ after $20 \mathrm{E}$ removal; in these times BHR4 transcripts increased; therefore, BHR4 is suggested to induce BFTZ-F1. Expression peak of $\beta F T Z-F 1$ was later than that of E74A and $\mathrm{BR}-\mathrm{C}$; therefore, E74A and BR-C are also induced by the remaining ecdysteroid in the hemolymph. BHR4 and EcR are suggested to function together to induce E74A and BR-C.

Induction of BR-C is inhibited by the addition of cycloheximide $6 \mathrm{~h}$ and $12 \mathrm{~h}$ after $20 \mathrm{E}$ removal; in these times BHR4 transcripts increased; therefore, BHR4 is suggested to induce BR-C. BR-C began to increase from V5 by the ecdysteroid in the hemolymph, since BR-C was inducible by the $0.02 \mu \mathrm{g} / \mathrm{ml} 20 \mathrm{E}$. Thereafter, its transcripts rapidly increased from the stage W3M, which is suggested to be induced by BHR4.

The results of ecdysone pulse treatment suggest that $B R-C, E 74 A$, and $\beta F T Z-F 1$ were induced by BHR4, and $B H R 4$ was induced by BHR3. Drosophila BHR3 homolog DHR3 repressed E74A (Lam et al., 1997), and DHR4 mutation reduced the expression of $E 74 A$ and $\beta F T Z-F 1$ (King-Jones et al., 2005). The present results corresponded well with these reports. Therefore, it is suggested that $E 74 A$ is inducible by ecdysone but repressed before W3L stage by BHR3 and induced by BHR4. The repression of $B R-C$ by BHR3 is suggested to be not strong as observed by Drosophila BR-C (Lam et al., 1997); therefore, $B R-C$ transcripts were detected from V5. Induction of BR-C by BHR4 was suggested in the present results. $\beta F T Z-F 1$ transcripts appeared earlier than $E 74 A$ transcripts, which is suggested that $\beta F T Z-F 1$ is not repressed but inducible by BHR3 as observed in Drosophila BFTZ-F1 (Lam et al., 1997; White et al., 1997). From the present results, it is suggested that
BHR3 induces BHR4 and $\beta F T Z-F 1$ but represses $E 74 A$ at W3E stage, and BHR4 induces E74A, BR-C, and $B F T Z$ $F 1$. This conclusion agrees well with above reports.

By the ecdysone surge, BHR3 is induced, and BHR3 regulates BHR4 and following ERTFs expression. Each ERTF regulate relating cuticular protein genes and other genes that function for the metamorphosis.

\section{Successive expression of ERTFs and their target genes}

Target genes of ERTFs have been reported previously; we have reported the possibility that ERTFs regulated $\mathrm{CP}$ genes and $\mathrm{CP}$ genes are expressed according to their regulating ERTFs respectively. The present paper showed 53 possible ERTF regulating $C P$ genes. Involvement of EcRE in Drosophila DDC gene promoter (Chen, Reece, O'Keefe, Engstrom, \& Hodgetts, 2002) and Aedes Vitellogenin gene (Martin, Wang, \& Raikhel, 2001). Target genes of E74A have been identified in Drosophila (L71-1, Fletcher, D'Avino, \& Thummel, 1997, L71-6, Urness, \& L., and Thummel, S. C., $1995)$ and those of $\beta F T Z-F 1$ were almost $C P$ genes (EDG84A, Murata et al., 1996) and (Bombyx; bmACP-6.7, Shiomi et al., 2000, Nita et al., 2009; Wang, Nita, et al., 2009). Most of BR-C genes functioned together with other ERTFs (Cakouros, Daish, Martin, Baehrecke, \& Kumar, 2002; BhC4-1, Basso Jr, Monesi, \& Paçó-Larson, 2006, DDC, Chen, O'Keefe, \& Hodgetts, 2002; Wang, Iwanaga, \& Kawasaki, 2009; Wang, Nita, et al., 2009). Thus, ERTFs regulate $\mathrm{CP}$ genes, and $\mathrm{CP}$ genes are expressed according to their regulating ERTFs respectively, as Weller et al. described Weller et al. (2001). The present paper showed 53 possible ERTF regulating CP genes. Thus, CP genes were successively expressed at the prepupal stage in wing discs of Bombyx mori.

\section{Conclusion}

According to regulating ERTFs, six different groups of $\mathrm{CP}$ genes expression pattern were observed. Expression timing of $\mathrm{CP}$ genes is regulated by ERTFs and the character of cuticle is determined by the combination of cuticular proteins. Group2, Group3, and Group4 include three $\mathrm{CPH}$ and six CPG genes. $\mathrm{CPH}$ and CPG have not $R \& R$ consensus and they are thought to be involved in epi- and procuticle layers. Among them Group4 genes are expressed from V5 stage; the reason is unclear. It is suggested that at least if involved in the pupal cuticle, they should be expressed after W2 stage, since BHR3 is the signal for the metamorphosis. From the present study, it is suggested that RR1 and RR2 cuticular protein genes are expressed at the stages W3L and P0 to construct procuticle, CPHs are from W2 to P0, CPGs are mainly from V5 to $\mathrm{P} 0$, and CPTs are from W3L to P0 stage. From this, it is suggested that CPR proteins 
construct procuticle and other CPs construct epi- and procuticle layers.

In the present paper, we offered a large number of $\mathrm{CP}$ genes as a candidate of ERTF-regulated genes and thus bring about insect metamorphosis. As described above, ERTFs are successively expressed and induce their target genes, which resulted in the metamorphosis.

\section{Supplementary information}

Supplementary information accompanies this paper at https://doi.org/10. 1186/s41936-019-0135-x.

Additional file 1: Figure S1. Developmental profile of Group3 CP gene $\mathrm{Bmor} C \mathrm{PH} 33$. Each datum was calculated from three independent experiments. The result is expressed as the mean \pm S. E. M. RNA was extracted from wing discs and reverse-transcribed to cDNA for use in qRT-PCR. Values were first normalized to $r p /$ and then plotted relative to the highest value for each panel. The highest expression level of each gene was designated as 1. Figure S2. Developmental profile of Group4 CP genes. Sample preparation and data treatment are the same as those in Figure S1. Figure S3. Developmental profile of Group5 CP genes. Sample preparation and data treatment are the same as those in Figure S1. Figure S4. Developmental profile of Group6 CP genes. Sample preparation and data treatment are the same as those in Figure S1. Figure S5. Effect of 20E addition. V4 wing discs were incubated for the indicated time in a medium containing $2 \mu \mathrm{g} / \mathrm{ml} 20 \mathrm{E}$ with (open circle) or without (closed circle) cycloheximide $(50 \mu \mathrm{g} / \mathrm{ml})$. RNA was extracted from wing discs and reverse-transcribed to CDNA for use in qRT-PCR. Values were first normalized to $\mathrm{rp} /$ and then plotted relative to the $0 \mathrm{~h}$ value for each panel. The $0 \mathrm{~h}$ expression level of each gene was designated as 0.1. Each datum was calculated from three independent experiments. The result is expressed as the mean \pm S. E. M. Asterisks indicate $p<0.05$ significance by the student's t-test. Level of mRNA of the ecdysone treatment. Figure S6. Effect of 20E addition of Group4 CP genes. Sample preparation and data treatment are the same as those in Figure S5. Figure S7. Effect of 20E addition of Group5 CP genes. Sample preparation and data treatment are the same as those in Figure S5. Figure S8. Developmental profile of Group5 CP genes that were up-regulated by the $20 \mathrm{E}$ addition. Sample preparation and data treatment are the same as those in Figure S5. Figure S9. Developmental profile of Group5 CP genes that were not up-regulated by the $20 \mathrm{E}$ addition. Sample preparation and data treatment are the same as those in Figure S5. Figure S10. Effect of ecdysone pulse treatment of Group3 gene. Wing discs of the W2 stage were incubated $12 \mathrm{~h}$ in a medium containing $2 \mu \mathrm{g} / \mathrm{ml} 20 \mathrm{E}$ and then transferred to a hormone-free medium with (open circle) or without cycloheximide $(50 \mu \mathrm{g} / \mathrm{ml})$ for the indicated time. RNA was extracted from wing discs and reverse-transcribed to CDNA for use in qRT-PCR. Values were first normalized to $\mathrm{rpl}$ and then plotted relative to the highest value for each panel. The highest expression level of each gene was designated as 1. Level of mRNA after ecdysone pulse treatment. Each datum was calculated from three independent experiments. The result is expressed as the mean $\pm S$. E. M. Asterisks indicate $p<0.05$ significance by the student's t-test. Level of mRNA of the ecdysone treatment. Figure S11. Effect of ecdysone pulse treatment of Group4 gene. Sample preparation and data treatment are the same as those in Figure S10. Figure S12. Effect of ecdysone pulse treatment of Group5 gene. Sample preparation and data treatment are the same as those in Figure S10. Figure S13. Effect of ecdysone pulse treatment of Group6 gene. Sample preparation and data treatment are the same as those in Figure S10.

Additional file 2: Table S1. List of primers.

Additional file 3: Table S2. Groups of CP genes and regulating ERTFs.

\section{Abbreviations}

20EA: 20E addition; 20EP: 20E pulse treatment; CP: Cuticular protein; CPFL: CPF-like; E74B: ETS transcription factor E74B; ERTF: Ecdysone responsive transcription factor

\section{Acknowledgements}

The author would like to thank MEXT: Japan Government for supporting the funds.

\section{Authors' contributions}

AMS, MB, SAH, and YM were responsible for suggesting, planning, designing the study, and writing the manuscript, and AMS was major in writing the manuscript. AMS performed experiments. All authors cooperate in revising the manuscript, read, and approved the final manuscript.

\section{Funding}

This work was partially supported by a grant-in-aid for scientific research from the Ministry of Education, Science, and Culture of Japan.

Availability of data and materials

All data are available upon request.

Ethics approval and consent to participate

Not applicable.

Consent for publication

Not applicable.

\section{Competing interests}

The authors declare that they have no competing interests.

\section{Author details}

${ }^{1}$ Department of Applied Biology, Kyoto Institute of Technology, Sakyo-ku, Kyoto 606-8585, Japan. ${ }^{2}$ Faculty of Agriculture, Bangladesh Jute Research Institute, Manik Mia Avenue, Dhaka 1207, Bangladesh. ${ }^{3}$ Department of Human Nutrition, Food and Animal Sciences, University of Hawaii at Manoa, Honolulu, HI, USA. ${ }^{4}$ Entomology Department, Bangabandhu Sheikh Mujibur Rahman Agricultural University, Gazipur 1706, Bangladesh.

Received: 11 June 2019 Accepted: 7 November 2019

Published online: 04 December 2019

\section{References}

Ali, M. S., Hossain, M. T., \& Mishra, B. (2018). Transcriptional profiling shows that BHR4 and E74A are the regulators of $\mathrm{CPH} 33$ and $\mathrm{CPH} 34$ gene expression. Journal of Advances in Molecular Biology, 2(1).

Ali, M. S., Iwanaga, M., \& Kawasaki, H. (2012). Ecdysone-responsive transcription factors determine the expression region of target cuticular protein genes in the epidermis of Bombyx mori. Development Genes and Evolution, 222, 89-97.

Ali, M. S., Iwanaga, M., \& Kawasaki, H. (2013). Ecdysone-responsive transcriptional regulation determines the temporal expression of cuticular protein genes in wing discs of Bombyx mori. Gene, 512, 337-347.

Ali, M. S., Mishra, B., Polan, M. S., Ninagi, O., Swapon, A. H., \& Yamaguchi, M. (2016). Regulation studies of a cuticle protein underlying genomic analysis. International Journal Molecular Genet Gene Ther, 1(2).

Ali, M. S., Mishra, B., Rahman, R. F., \& Swapon, A. H. (2016). The silkworm Bombyx mori cuticular protein CPR55 gene is regulated by the transcription factor BFTZ-F1. The Journal of Basic \& Applied Zoology, 73, 20-27.

Ali, M. S., Rahman, R. F., \& Swapon, A. H. (2015). Transcriptional regulation of cuticular protein glycine-rich13 gene expression in wing disc of Bombyx mori. Lepidoptera J. Insect Sci., 27, 1-6.

Ali, M. S., Wang, H. B., Iwanaga, M., \& Kawasaki, H. (2012). Expression of cuticular protein genes, BmorCPG11 and BMWCP5 is differently regulated at the prepupal stage in wing discs of Bombyx mori. Com. Biochemistry and Physiology. Part B, 162, 44-50.

Andersen, S. O. (1998). Amino acid sequence studies on endocuticular proteins from the desert locust, Schistocerca gregaria. Insect Biochemistry and Molecular Biology, 28, 421-434.

Andersen, S.O. (2000). Studies on proteins in post-ecdysial nymphal cuticle of locust, Locusta migratoria, and cockroach, Blaberus cranifer. Insect Biochemistry and Molecular Biology. 30, 569-577.

Andersen, S. O., Rafn, K., \& Roepstorff, P. (1997). Sequence studies of proteins from klarval and pupal cuticle of the yellow mealworm, Tenebrio moliter. Insect Biochemistry and Molecular Biology, 27, 121-131. 
Basso, L.R Jr, de C Neves M, Monesi, N., Paçó-Larson M.L. (2006). Broad -Complex, E74 and E75 early genes control DNA puff BhC4-1 expression in prepupal salivary gland. Genesis 44, 505-514.

Bayer, C., Zhou, X., Zhou, B., Riddiford, L. M., \& von Kalm, L. (2003). Evolution of the Drosophila broad locus: the Manduca sexta broad Z4 isoform has biological activity in Drosophila. Development Genes and Evolution, 213, 471-476.

Cakouros, D., Daish, T., Martin, D., Baehrecke, E. H., \& Kumar, S. (2002). Ecdysone induced expression of the caspase DRONC during hormone dependent programmed cell death in Drosophila is regulated by Broad-Complex. The Journal of Cell Biology, 157, 985-995.

Charles, J. P. (2010). The regulation of expression of insect cuticle protein genes. Insect Biochemistry and Molecular Biology, 40, 205-213.

Chen, L., O'Keefe, S. L., \& Hodgetts, R. B. (2002). Control of Dopa decarboxylase gene expression by the Broad-Complex during metamorphosis in Drosophila. Mechanisms of Development, 119, 145-156.

Chen, L., Reece C., O'Keefe SL, Hawryluk, G.W.L, Engstrom, M.M., Hodgetts RB (2002). Induction of the early-late Ddc gene during Drosophila metamorphosis by the ecdysone receptor. Mechanisms of Development 114 95-107.

Chen, L., Zhu, J., Sun, G., \& Raikhel, A. S. (2004). The early gene Broad is involved in the ecdysteroid hierarchy govering vitellogenesis of the mosquito Aedes aegypti. Journal of Molecular Endocrinology, 33, 743-761.

Cornman, R. S., Togawa, T., Dunn, W. A., He, N., Emmons, A. C., \& Willis, J. H. (2008). Annotation and analysis of a large cuticular protein family with the R\&R Consensus in Anopheles gambiae. BMC Genomics, 18, 9-22.

Dittmer, N. T., Tetreau, G., Cao, X., Jiang, H., Wang, P., \& Kanost, M. R. (2015). Annotation and expression analysis of cuticular proteins from the tobacco hornworm, Manduca sexta. Insect Biochemistry and Molecular Biology, 62, $100-113$.

Elgendy, A. M., Tufail, M., Mohamed, A. A., \& Takeda, M. (2019). A putative direct repeat element plays a dual role in the induction and repression of insect vitellogenin-1 gene expression. Comparative Biochemistry and Physiology Part B: Biochemistry and Molecular Biology, 234, 1-8.

Fletcher, J. C., D'Avino, P. P., \& Thummel, C. S. (1997). A steroid-trigerred switch in E74 transcription factor isoforms regulates the timing of secondary-response gene expression.

Fletcher, J. C., \& Thummel, C. S. (1995). The ecdysone-inducible Broad-Complex and E74 early genes interact to regulate target gene transcription and Drosophila metamorphosis. Genetics, 141, 1025-1035.

Futahashi, R., Okamoto, S., Kawasaki, H., Zhong, Y. S., Iwanaga, M., Mita, K., \& Fujiwara, H. (2008). Genome-wide identification of cuticular protein genes in the silkworm, Bombyx mori. Insect Biochemistry and Molecular Biology, 38, $1138-1146$

Guan, X., Middlebrooks, B. W., Alexander, S., \& Wasserman, S. A. (2006). Mutation of TweedleD, a member of an unconventional cuticle protein family, alters body shape in Drosophila. Proceedings of the National Academy of Sciences of the United States of America, 103, 16794-16799.

Hiruma, K., \& Riddiford, L. M. (2001). Regulation of transcription factors MHR4 and BFTZ-F1 by 20-hydroxyecdysone during a larval molt in the tobacco hornworm, Manduca sexta. Developmental Biology, 232, 265-274.

Huet, F., Ruiz, C., \& Richards, G. (1995). Sequential gene activation by ecdysone in Drosophila melanogaster: the hierarchical equivalence of early and early late genes. Development, 121, 1195-1204.

Kawasaki, H. (1989). Methods for culture of Bombyx mori wing discs. Journal of Tissue Culture Methods, 12, 31-33.

Kawasaki, H., Hirose, S., \& Ueda, H. (2002). BFTZ-F1 dependent and independent activation of Edg78E, a pupal cuticle gene, during the early metamorphic period in Drosophila melanogaster. Development, Growth \& Differentiation, 44, 419-425.

King-Jones, K., Charles, J.-P., Lam, G., \& Thummel, S. C. (2005). The ecdysoneinduced DHR4 orphan nuclear receptor coordinates growth and maturation in Drosophila. Cell, 121, 773-784.

Konopova, B., \& Jindra, M. (2008). Broad-Complex acts downstream of Met in juvenile hormone signaling to coordinate primitive holometabolan metamorphosis. Development, 135, 559-568.

Koyama, T., Syropyatova, M. O., \& Riddiford, L. M. (2008). Insulin/IGF signaling regulates the change in commitment in imaginal discs and primodia by overriding the effect of juvenile hormone. Developmental Biology., 324, 258-256.

Lam, T. G., Bonnie, L. H., Bender, M., \& Thummel, C. S. (1999). DHR3 is required for the prepupal-pupal transition and differentiation of adult structures during Drosophila metamorphosis. Developmental Biology, 212, 204-216.
Lam, T. G., Jiang, C., \& Thummel, C. S. (1997). Coordination of larval and prepupal gene expression by DHR3 orphan receptor during Drosophila metamorphosis. Development, 124, 1757-1769.

Liang, J., Zhang, L., Xiang, Z., \& He, N. (2010). Expession profile of cuticular genes of silkworm, Bombyx mori. BMC Genomics, 11, 173.

Martin, D., Wang, S. F., \& Raikhel, A. S. (2001). The vitellogenin gene of the mosquito Aedes aegypti is a direct target of ecdysteroid receptor. Insect Biochemistry and Molecular Biology, 31, 827-837.

Muramatsu, D., Kinjoh, T., Shinoda, T., \& Hiruma, K. (2008). The role of 20hydroxyecdysone and juvenile hormone in pupal commitment of the epidermis of the silkworm, Bombyx mori. Mechanisms of Development, 125, 411-420.

Murata, T., Kageyama, Y., \& Hirose, S. (1996). Regulation of the EDG84A gene by FTZ-F1 during metamorphosis in Drosophila malanogaster. Molecular and Cellular Biology, 16, 6509-6515.

Nita, M., Wang, H. B., Zhong, Y. S., Mita, K., Iwanaga, M., \& Kawasaki, H. (2009). Analysis of ecdysone-pulse responsive region of $B M W C P 2$ in wing disc of Bombyx mori. Com Biochem Physiol Part B, 153, 101-108.

Okamoto, S., Futahashi, R., Kojima, T., Mita, K., \& Fujiwara, H. (2008). Catalogue of epidermal genes expressed in the epidermis during larval molt of the silkworm Bombyx mori. BMC Genomics, 9, 396.

Pan, P. L., Ye, Y. X., Lou, Y. H., Lu, J. B., Cheng, C., Shen, Y., \& Zhang, C. X. (2018). A comprehensive omics analysis and functional survey of cuticular proteins in the brown planthopper. Proceedings of the National Academy of Sciences, 115(20), 5175-5180.

Parthasarathy, R., Tan, A., Bai, H., \& Palli, S. R. (2008). Transcription factor broad suppresses precocious development of adult structures during larval-pupal metamorphosis in the red flour beetle, Tribolium castaneum. Mech Dev, 125, 299-313.

Riddiford, L. M., Hiruma, K., Zhou, X., \& Nelson, C. A. (2003). Insights into the molecular basis of the hormonal control of molting and metamorphosis from Manduca sexta and Drosophila melanogaster. Insect Biochemistry and Molecular Biology, 33, 1327-1338.

Riddiford, L. M., \& Truman, J. W. (1993). Hormone receptors and the regulation of insect metamorphosis. American Zoologist, 33, 340-347.

Sakurai, S., Kaya, M., \& Satake, S. (1998). Hemolymph ecdysteroid titer and ecdysteroid-dependent development events in the last-larval stadium of the silkworm, Bombyx mori: role of low cdysteroid titer in larval-pupal metamorphosis and a reappraisal of the head critical period. Journal of Insect Physiology, 44, 867-881.

Sekimoto, T., Iwami, M., \& Sakurai, S. (2006). Coordinate responses of transcription factors to ecdysone during programmed cell death in the anterior silk gland of the silkworm, Bombyx mori. Insect Molecular Biology, 15, 281-292.

Sekimoto, T., Iwami, M., \& Sakurai, S. (2007). 20-hydroxyecdysone regulation of two isoforms of the Ets transcription factor E74 gene in programmed cell death in the silkworm anterior silk gland. Insect Molecular Biology, 16 581-590.

Shiomi, K., Niimi, T., Imai, K., \& Yamashita, O. (2000). Structure of the VAPpeptide (BmACP-6.7) gene in the silkworm, Bombyx mori and a possible regulation of its expression by $\beta F T Z-F 1$. Insect Biochemistry and Molecular Biology, 30, 119-125.

Stilwell, G. E., Nelson, C. A., Weler, J., Cui, H., Hiruma, K., Truman, J. W., \& Riddiford, L. M. (2003). E74 exhibits stage-specific hormonal regulation in the epidermis of the tobocco hornworm, Manduca sexta. Developmental Biology, 258, 76-90.

Sun, G., Zhu, J., Chen, L., \& Raikhel, A. S. (2005). Synergistic action of E74B and ecdysteroid receptor in activating 20-hydroxyecdysone effector gene. Proceedings of the National Academy of Sciences of the United States of America, 102, 15506-15511.

Sun, G.-C., Hirose, S., \& Ueda, H. (1994). Intermittent expression of BmFTZ-F1, a member of the nuclear hormone receptor superfamilyduring development of the silkworm Bombyx mori. Developmental Biology, 162, 426-437.

Thummel, C., \& S. (1995). From embryogenesis to metamorphosis: The regulation and function of Drosophila nuclear receptor superfamily members. Cell, 83, 871-877.

Togawa, T., Dunn, W. A., Emmons, A. C., \& Willis, J. H. (2007). CPF and CPFL, two related gene families encoding cuticular proteins of Anopheles gambiae and other insects. Insect Biochemistry and Molecular Biology, 37, 675-688.

Togawa, T., Dunn, W. A., Emmons, A. C., Nagao, J., \& Willis J. H. (2008). Developmental expression patterns of cuticular protein genes with the $R \& R$ Consensus from Anopheles gambiae. Insect Biochemistry and Molecular Biology, 38, 508-519. 
Togawa, T., Natkato, H., \& Izumi, S. (2004). Analysis of the chitin recognition mechanim of cuticle protins from the soft cuticle of the silkworm, Bombyx mori. Insect Biochemistry and Molecular Biology, 34, 1059-1067.

Uhlirova, M., Foy, B. D., Beaty, B. J., Olson, K. E., Riddiford, L. M., \& Jindra, M. (2003). Use of sindbis virus-mediated RNA interference to demonstrate a conserved role of Broar-Complex in insect metamorhosis. Proceedings of the National Academy of Sciences of the United States of America, 100, 15607-15612.

Urness, D. L., \& Thummel, S. C. (1995). Molecular analysis of a steroid-induced regulatory hierarchy: The Drosophila E74A protein directly regulates L71-6 transcription. The EMBO Journal, 14, 6239-6246.

Wang, H. B., Iwanaga, M., \& Kawasaki, H. (2009). Activation of BMWCP10 promoter and regulation by BR-C Z2 in wing disc of Bombyx mori. Insect Biochemistry and Molecular Biology, 39, 615-623.

Wang, H. B., Moriyama, M., Iwanaga, M., \& Kawasaki, H. (2010). Ecdysone directly and indirectly regulates a cuticle protein gene, BMWCP10, in the wing disc of Bombyx mori. Insect Biochemistry and Molecular Biology, 40, 453-459.

Wang, H. B., Nita, M., Iwanaga, M., \& Kawasaki, H. (2009). BFTZ-F1 and BroadComplex positively regulate the transcription of the wing cuticle protein gene, BMWCP5, in wing discs of Bombyx mori. Insect Biochemistry and Molecular Biology, 39, 624-633.

Weller, J., Sun, G.-C., Zhou, B., Lan, Q., Hiruma, K., \& Riddiford, L. M. (2001). Isolationand developmental expression of two nuclear receptors, MHR4 and BFTZ-F1, in the tobacco hornworm, Manduca sexta. Insect Biochemistry and Molecular Biology, 31, 827-837.

White, K. P., Hurban, P., Watanabe, T., \& Hogness, D. S. (1997). Coordination of Drosophila metamorphosis by two ecdysone-induced nuclear receptors. Science, 276, 114-117.

Wigglesworth, V. B. (1972). The Integment. Chapter II in The principles of insect physiology', (7th ed., ). London: Chapman and Hall.

Willis, J. H. (2010). Structurl cuticular proteis from arthropods: annotation nomenclature, and sequence characteristics in genomics era. Insect Biochemistry and Molecular Biology, 40, 241-251.

Zhao, X., Gou, X., Qin, Z., Li, D., Wang, Y., Ma, E., \& Zhang, J. (2017). Identification and expression of cuticular protein genes based on Locusta migratoria transcriptome. Scientific Reports, 7, 45462.

Zhou, B., \& Riddiford, L. M. (2001). Hormonal regulation and patterning of the Broad-Complex in the epidermis and wing discs of the tobacco hornworm, Manduca sexta. Developmental Biology, 231, 125-137.

Zhou, X., \& Riddiford, L. M. (2002). Broad specifies pupal development and mediates the "status quo" action of juvenile hormone on the pupal-adult transformation in Drosophila and Manduca. Development., 129, 2259-2269.

\section{Publisher's Note}

Springer Nature remains neutral with regard to jurisdictional claims in published maps and institutional affiliations.

\section{Submit your manuscript to a SpringerOpen ${ }^{\circ}$ journal and benefit from:}

- Convenient online submission

- Rigorous peer review

- Open access: articles freely available online

- High visibility within the field

- Retaining the copyright to your article

Submit your next manuscript at $\boldsymbol{\nabla}$ springeropen.com 